\title{
Shock wave structure of multi-temperature Euler equations from kinetic theory for a binary mixture
}

\author{
Marzia Bisi Giorgio Martalò Giampiero Spiga
}

\begin{abstract}
A multi-temperature hydrodynamic limit of kinetic equations is employed for the analysis of the steady shock problem in a binary mixture. Numerical results for varying parameters indicate possible occurrence of either smooth profiles or of weak solutions with one or two discontinuities.
\end{abstract}

\section{Introduction}

Multi-temperature fluid-dynamic models are being quite widely studied in modeling and investigating gas mixtures, either inert or reactive $[10,2]$. They give rise typically to hyperbolic systems of balance laws which can be studied in the frame of extended thermodynamics [9]. An interesting point in this respect is a comparison with the derivation of fluid-dynamic equations of this type, with momentum and energy exchange rates, as suitable hydrodynamic limit, starting from a kinetic theory description $[7,1]$. Indeed this paper belongs to the latter research line, and is aimed at testing a simple fluid-dynamic model at Euler level, derived from the relevant Boltzmann equations, on a very classical problem like the one dimensional steady shock waves in a binary mixture. The shock problem has also been very extensively investigated, and significant results are available on occurrence of a smooth solution or on the presence of discontinuities (so called subshocks) $[3,5]$.

The present multi-temperature and multi-velocity model allows an explicit representation of all exchange terms. Results, still on preliminary stage, are in agreement with those established in the fluid-dynamic literature, and show, even for such a simple physical scenario, possible occurrence of interesting phenomena like sub-shocks of different kinds and weak solutions with more than one discontinuity.

Specifically, we consider a mixture of two mono-atomic and rarefied gases $A^{1}, A^{2}$, whose particles, of mass $m^{1}$ and $m^{2}$, interact by means of elastic collisions. We assume that the dominant process in the evolution is given by elastic encounters between particles of the same species, i.e. we consider the following re-scaled Boltzmann equations [4]

$$
\begin{aligned}
& \frac{\partial f^{1}}{\partial t}+\mathbf{v} \cdot \nabla_{\mathbf{x}} f^{1}=\frac{1}{\varepsilon} Q^{11}\left(f^{1}, f^{1}\right)+Q^{12}\left(f^{1}, f^{2}\right) \\
& \frac{\partial f^{2}}{\partial t}+\mathbf{v} \cdot \nabla_{\mathbf{x}} f^{2}=Q^{21}\left(f^{2}, f^{1}\right)+\frac{1}{\varepsilon} Q^{22}\left(f^{2}, f^{2}\right),
\end{aligned}
$$


where $f^{1}, f^{2}$ are the distribution functions of gases $A^{1}, A^{2}$, respectively, $Q^{s r}$ is the Boltzmann collision operator describing effects due to collisions between particles of species $s$ and $r$, with $s, r=1,2$, and the small parameter $\varepsilon$ stands for the Knudsen number. We are concerned here with the corresponding fluid-dynamic equations at Euler level, obtained by closing the macroscopic "conservation" equations relevant to the dominant operator only. In case of one space dimension, to which we will stick here, we have thus to deal with 6 evolution equations for 6 unknown fields, number densities $n^{1}, n^{2}$, mass velocities $u^{1}, u^{2}$, and species temperatures $T^{1}, T^{2}$. It is well known that collision contributions arise on the right hand sides due to exchange of momentum and energy in the slow scattering process involving particles $\left(A^{1}, A^{2}\right)$, and that they are provided by suitable weak forms of the elastic Boltzmann operators $Q^{12}$ or $Q^{21}$. For the momentum and energy production rates of species one, labeled by $R_{12}$ and $S_{12}$ respectively, under the simplifying assumption of Maxwell molecule interactions [4], we have the explicit expressions [2]

$$
\begin{gathered}
R_{12}=-\mu_{12} n^{1} n^{2} \bar{\nu}_{12}\left(u^{1}-u^{2}\right), \\
S_{12}=-\mu_{12} n^{1} n^{2} \bar{\nu}_{12}\left[\left(\alpha_{12} u^{1}+\alpha_{21} u^{2}\right)\left(u^{1}-u^{2}\right)+3 \frac{T^{1}-T^{2}}{m^{1}+m^{2}}\right],
\end{gathered}
$$

where $\mu^{12}=m^{1} m^{2} /\left(m^{1}+m^{2}\right)$ is the reduced mass, $\alpha_{12}=\mu^{12} / m^{2}$ and $\alpha_{21}=\mu^{12} / m^{1}$ are mass ratios, and $\bar{\nu}_{12}$ is a suitable (constant) collision frequency.

The equations for the steady shock problem are analyzed in the next section, going through the relevant dynamical system, the Rankine-Hugoniot conditions, and the possible singularities. The different scenarios arising for varying Mach number and upstream conditions are discussed in the following section, with reference to the pertinent jump conditions and stability properties of the limiting equilibrium states. The article is completed by a sample of numerical results on the shock profiles and on the development of different types of singularities when parameters are varied.

\section{Shock wave structure}

We shall test the multi-temperature and multi-velocity Euler equations deduced from kinetic theory on the classical steady shock problem, in which case the 6 observable fields are governed by the set of ordinary differential equations

$$
\begin{aligned}
\frac{d}{d x}\left(n^{1} u^{1}\right) & =0 \\
\frac{d}{d x}\left(n^{2} u^{2}\right) & =0 \\
\frac{d}{d x}\left[\rho^{1}\left(u^{1}\right)^{2}+n^{1} T^{1}\right] & =R_{12} \\
\frac{d}{d x}\left[\rho^{2}\left(u^{2}\right)^{2}+n^{2} T^{2}\right] & =-R_{12} \\
\frac{d}{d x}\left[\frac{1}{2} \rho^{1}\left(u^{1}\right)^{3}+\frac{5}{2} n^{1} T^{1} u^{1}\right] & =S_{12} \\
\frac{d}{d x}\left[\frac{1}{2} \rho^{2}\left(u^{2}\right)^{3}+\frac{5}{2} n^{2} T^{2} u^{2}\right] & =-S_{12}
\end{aligned}
$$


where $\rho^{s}=m^{s} n^{s}$. The shock solution must join two limiting equilibrium states at $x \rightarrow$ $\pm \infty$, which are characterized by a common mass velocity $u_{ \pm}^{1}=u_{ \pm}^{2}=u_{ \pm}$and a common temperature $T_{ \pm}^{1}=T_{ \pm}^{2}=T_{ \pm}$. Each state is then determined once we fix single number densities $n_{ \pm}^{1}, n_{ \pm}^{2}$, velocity $u_{ \pm}$, and temperature $T_{ \pm}$. These parameters define also the relevant sound speeds $c_{ \pm}$and the corresponding Mach numbers

$$
\mathrm{Ma}_{ \pm}^{2}=\frac{u_{ \pm}^{2}}{c_{ \pm}^{2}}=\frac{3 \rho_{ \pm} u_{ \pm}^{2}}{5 n_{ \pm} T_{ \pm}},
$$

where $n_{ \pm}=n_{ \pm}^{1}+n_{ \pm}^{2}$ and $\rho_{ \pm}=\rho_{ \pm}^{1}+\rho_{ \pm}^{2}$. From system (4) we may deduce four independent conservation laws: the first two equations, the sum of third and fourth equation (momentum conservation), the sum of fifth and sixth equation (energy conservation). These four conservations establish four relations between downstream $(+)$ and upstream (-) parameters, the so-called Rankine-Hugoniot conditions:

$$
\begin{aligned}
n_{+}^{s} u_{+} & =n_{-}^{s} u_{-}, \quad s=1,2 \\
\rho_{+}\left(u_{+}\right)^{2}+n_{+} T_{+} & =\rho_{-}\left(u_{-}\right)^{2}+n_{-} T_{-}=: \kappa^{A} \\
\rho_{+}\left(u_{+}\right)^{3}+5 n_{+} T_{+} u_{+} & =\rho_{-}\left(u_{-}\right)^{3}+5 n_{-} T_{-} u_{-}=: \kappa^{B} .
\end{aligned}
$$

These allow to express the downstream parameters as functions of the upstream ones (and of the upstream Mach number $\mathrm{Ma}_{-}$) [6]:

$$
\begin{aligned}
& n_{+}^{s}=\frac{4 \mathrm{Ma}_{-}^{2}}{\mathrm{Ma}_{-}^{2}+3} n_{-}^{s}, \quad s=1,2, \quad u_{+}=\frac{\mathrm{Ma}_{-}^{2}+3}{4 \mathrm{Ma}_{-}^{2}} u_{-} \\
& T_{+}=\frac{\left(\mathrm{Ma}_{-}^{2}+3\right)\left(5 \mathrm{Ma}_{-}^{2}-1\right)}{16 \mathrm{Ma}_{-}^{2}} T_{-} .
\end{aligned}
$$

Moreover, conservations following from system (4) imply that, if we fix the upstream configuration $n_{-}^{1}, n_{-}^{2}, u_{-}^{1}=u_{-}^{2}=u_{-}, T_{-}^{1}=T_{-}^{2}=T_{-}$, all points of the shock profile fulfill the constraints

$$
\begin{aligned}
n^{1} u^{1} & =n_{-}^{1} u_{-} \\
n^{2} u^{2} & =n_{-}^{2} u_{-} \\
\rho^{1}\left(u^{1}\right)^{2}+n^{1} T^{1}+\rho^{2}\left(u^{2}\right)^{2}+n^{2} T^{2} & =\rho_{-}\left(u_{-}\right)^{2}+n_{-} T_{-} \\
\rho^{1}\left(u^{1}\right)^{3}+5 n^{1} T^{1} u^{1}+\rho^{2}\left(u^{2}\right)^{3}+5 n^{2} T^{2} u^{2} & =\rho_{-}\left(u_{-}\right)^{3}+5 n_{-} T_{-} u_{-} .
\end{aligned}
$$

These four relations should allow to express four of our six unknown fields in terms of the remaining two. The first two equations yield $n^{1}=n_{-}^{1} u_{-} / u^{1}$ and $n^{2}=n_{-}^{2} u_{-} / u^{2}$. Then, third and fourth conservations may be seen as a linear system for the two temperatures $T^{1}, T^{2}$, that, if it is non singular, gives temperatures as functions of $u^{1}, u^{2}$. Unfortunately, the determinant of coefficient matrix is $5 n^{1} n^{2}\left(u^{2}-u^{1}\right)$ hence it vanishes for $u^{1}=u^{2}$. This is a problem because we want to determine an heteroclinic orbit joining two equilibrium states, in which of course $u^{1}=u^{2}$. More precisely, the four conditions (8) are not capable to reproduce correctly the equilibrium states; in fact, if we have $u^{1}=u^{2}=u_{-}$, the first two equations correctly give $n^{1}=n_{-}^{1}$ and $n^{2}=n_{-}^{2}$, but third and fourth equations reduce to 
the unique condition $n_{-}^{1} T^{1}+n_{-}^{2} T^{2}=n_{-} T_{-}$, that of course does not imply $T^{1}=T^{2}=T_{-}$ (this result could be obtained only with the additional requirement $T^{1}=T^{2}$ ).

For these reasons we will resort to a different strategy, that will lead us to study a system of three ordinary differential equations. Other strategies can be devised, of course; the one that we shall follow here is probably the simplest for motivation and machinery. We introduce the new variables $\hat{T}=\left(n^{1} / n\right) T^{1}+\left(n^{2} / n\right) T^{2}$ and $\theta=T^{1}-T^{2}$ in place of temperatures $T^{1}, T^{2}$, from which

$$
T^{1}=\hat{T}+\frac{n^{2}}{n} \theta \quad \text { and } \quad T^{2}=\hat{T}-\frac{n^{1}}{n} \theta .
$$

We use the first three conservations (8) to obtain the three unknowns $n^{1}, n^{2}, \hat{T}$ in terms of the remaining ones $u^{1}, u^{2}, \theta$. Last conservation law in (8) will be controlled later. We avoid in this way the previous singular coupling between momentum and energy conservations, and discard the most cumbersome energy algebraic equation. Consequently, one differential equation suffices for momentum balance, whereas both differential equations for energy have to be retained. Having that in mind, we get

$$
n^{s}=\frac{n_{-}^{s} u_{-}}{u^{s}}, s=1,2 \quad \hat{T}=\frac{u^{1} u^{2} \kappa^{A}}{\left(n_{-}^{1} u^{2}+n_{-}^{2} u^{1}\right) u_{-}}-\frac{\left(\rho_{-}^{1} u^{1}+\rho_{-}^{2} u^{2}\right) u^{1} u^{2}}{n_{-}^{1} u^{2}+n_{-}^{2} u^{1}} .
$$

Now rewriting the problem in terms of $u^{1}, u^{2}, \theta$ is only matter of some patient and lengthy manipulations. Skipping all details, the subsystem of ODEs (4) made by one balance equation for momentum (for instance, third equation in (4)) and by the two balance equations for energy (last two equations in (4)) may be cast as $\mathbf{A} \cdot \frac{d \mathbf{y}}{d x}=\mathbf{b}$, where $\mathbf{y}=\left(u^{1}, u^{2}, \theta\right)^{T}, \mathbf{b}=\left(R_{12}, S_{12},-S_{12}\right)^{T}$, and the coefficient matrix $\mathbf{A}$ has the following entries:

$$
\left[\begin{array}{ccc}
\frac{n^{2}}{n u^{1}}\left[\rho^{1}\left(u^{1}\right)^{2}-n^{1} T^{1}\right] & -\frac{n^{1}}{n u^{2}}\left[\rho^{2}\left(u^{2}\right)^{2}-n^{2} T^{2}\right] & \frac{n^{1} n^{2}}{n} \\
\rho^{1}\left(u^{1}\right)^{2}-\frac{5}{2} \frac{n^{1}}{n}\left[\rho^{1}\left(u^{1}\right)^{2}-n^{1} T^{1}\right] & -\frac{5}{2} \frac{n^{1} u^{1}}{n u^{2}}\left[\rho^{2}\left(u^{2}\right)^{2}-n^{2} T^{2}\right] & \frac{5}{2} \frac{n^{1} n^{2} u^{1}}{n} \\
-\frac{5}{2} \frac{n^{2} u^{2}}{n u^{1}}\left[\rho^{1}\left(u^{1}\right)^{2}-n^{1} T^{1}\right] & \rho^{2}\left(u^{2}\right)^{2}-\frac{5}{2} \frac{n^{2}}{n}\left[\rho^{2}\left(u^{2}\right)^{2}-n^{2} T^{2}\right] & -\frac{5}{2} \frac{n^{1} n^{2} u^{2}}{n}
\end{array}\right]
$$

The determinant of matrix A turns out to be

$$
\operatorname{det}(\mathbf{A})=\frac{1}{4} \frac{n^{1} n^{2}}{n}\left[3 \rho^{1}\left(u^{1}\right)^{2}-5 n^{1} T^{1}\right]\left[3 \rho^{2}\left(u^{2}\right)^{2}-5 n^{2} T^{2}\right]
$$

and it vanishes if $\left(\mathrm{M}^{1}\right)^{2}=1$ or $\left(\mathrm{M}^{2}\right)^{2}=1$, where $\left(\mathrm{M}^{s}\right)^{2}=3 \rho^{s}\left(u^{s}\right)^{2} /\left(5 n^{s} T^{s}\right)$ denotes the Mach number we would have if we considered the evolution of species $A^{s}$ only.

In all points in which matrix $\mathbf{A}$ is regular, we may compute the inverse matrix $\mathbf{A}^{-1}$ that allows to write the system of ODEs in normal form. Skipping again technical details, 
the final result is

$$
\begin{aligned}
\frac{d u^{s}}{d x} & =\frac{5 R_{12} u^{s}-2 S_{12}}{3 \rho^{s}\left(u^{s}\right)^{2}-5 n^{s} T^{s}}, \quad s=1,2 \\
\frac{d \theta}{d x} & =-2 \sum_{s=1}^{2} \frac{R_{12} \rho^{s}\left(u^{s}\right)^{3}-S_{12}\left[\rho^{s}\left(u^{s}\right)^{2}-n^{s} T^{s}\right]}{n^{s} u^{s}\left[3 \rho^{s}\left(u^{s}\right)^{2}-5 n^{s} T^{s}\right]}
\end{aligned}
$$

where $n^{1}, n^{2}, T^{1}, T^{2}$ are explicit functions of the unknown fields $u^{1}, u^{2}, \theta$ by means of (9)-(10), and $R_{12}, S_{12}$ are collision contributions given in (2)-(3). Notice that the right hand sides of equations (13) vanish when $u^{1}=u^{2}$ and $\theta=0$, and in the particular cases $u^{1}=u^{2}=u_{ \pm}$(with $\theta=0$ ) formulas (9)-(10) correctly reproduce $n^{1}=n_{ \pm}^{1}, n^{2}=$ $n_{ \pm}^{2}, T^{1}=T^{2}=T_{ \pm}$. It can be checked by lengthy direct computations that a suitable combination of equations (13) implies that also last conservation law in (8) holds, i.e. $\rho^{1}\left(u^{1}\right)^{3}+5 n^{1} T^{1} u^{1}+\rho^{2}\left(u^{2}\right)^{3}+5 n^{2} T^{2} u^{2}=\kappa^{B}$.

Possible smooth shock wave solutions to the system (13) may exist only if denominators

$$
\mathcal{D}^{s}=3 \rho^{s}\left(u^{s}\right)^{2}-5 n^{s} T^{s}=5 n^{s} T^{s}\left[\left(\mathrm{M}^{s}\right)^{2}-1\right], \quad s=1,2
$$

do not vanish during the evolution. We may evaluate such terms in the upstream and downstream equilibria. Introducing concentration $c=n_{-}^{1} / n_{-}\left(\equiv c_{-}\right)$and the mass ratio $\alpha=m^{1} / m^{2}$, and bearing in mind the definition of Mach numbers (5) we have

$$
\mathrm{Ma}_{-}^{2}=c\left(\mathrm{M}_{-}^{1}\right)^{2}+(1-c)\left(\mathrm{M}_{-}^{2}\right)^{2}, \quad\left(\mathrm{M}_{-}^{1}\right)^{2}=\alpha\left(\mathrm{M}_{-}^{2}\right)^{2},
$$

hence, if $c$ and $\alpha$ are fixed, $\left(\mathbf{M}_{-}^{1}\right)^{2}$ and $\left(\mathbf{M}_{-}^{2}\right)^{2}$ are completely determined in terms of $\mathbf{M a}_{-}^{2}$ :

$$
\left(\mathrm{M}_{-}^{1}\right)^{2}=\frac{\alpha}{\gamma} \mathrm{Ma}_{-}^{2}, \quad\left(\mathrm{M}_{-}^{2}\right)^{2}=\frac{1}{\gamma} \mathrm{Ma}_{-}^{2}, \quad \gamma=\alpha c+1-c .
$$

From now on let us assume $\alpha<1$, but analogous considerations would hold of course also for $\alpha>1$. Under this option we have immediately $\mathrm{M}_{-}^{1}<\mathrm{M}_{-}^{2}$ and $\alpha<\gamma<1$. It is well known [6] that the entropy flux condition imposed by the $H$-theorem implies the constraint $\mathrm{Ma}_{-}>1$ for existence of a shock wave. Hence, since $\mathrm{Ma}_{-}^{2}$ is a suitably weighted mean of $\left(\mathrm{M}_{-}^{1}\right)^{2}$ and of $\left(\mathrm{M}_{-}^{2}\right)^{2}$, at least the greatest of the two species upstream Mach numbers $\mathrm{M}_{-}^{s}$ will be greater than 1 . As concerns the same Mach numbers in the downstream equilibrium, it is not difficult to show that, owing to (16) and to relations (7), we have

$$
\left(\mathrm{M}_{+}^{1}\right)^{2}=\frac{\alpha}{\gamma} \frac{\mathrm{Ma}_{-}^{2}+3}{5 \mathrm{Ma}_{-}^{2}-1} \quad\left(\mathrm{M}_{+}^{2}\right)^{2}=\frac{1}{\gamma} \frac{\mathrm{Ma}_{-}^{2}+3}{5 \mathrm{Ma}_{-}^{2}-1} .
$$

Once the configuration in the upstream equilibrium is fixed, for $\mathrm{Ma}_{-}=1$ we have $\left(\mathrm{M}_{-}^{1}\right)^{2}=$ $\left(\mathrm{M}_{+}^{1}\right)^{2}=\alpha / \gamma, \quad\left(\mathrm{M}_{-}^{2}\right)^{2}=\left(\mathrm{M}_{+}^{2}\right)^{2}=1 / \gamma$; if now we vary Mach number $\mathrm{Ma}_{-}^{2}$ from 1 to $+\infty$ keeping fixed the concentration $c$ (hence the parameter $\gamma$ ), then $\left(\mathrm{M}_{-}^{1}\right)^{2}$ and $\left(\mathrm{M}_{-}^{2}\right)^{2}$ will linearly increase up to $+\infty$, while $\left(\mathrm{M}_{+}^{1}\right)^{2}$ and $\left(\mathrm{M}_{+}^{2}\right)^{2}$ will decrease up to the limiting values $\alpha /(5 \gamma), 1 /(5 \gamma)$, respectively.

It is easy to see that $\mathcal{D}_{+}^{1}<0$ and $\mathcal{D}_{-}^{2}>0$ for any $\mathrm{Ma}_{-} \geq 1$. Existence of a smooth shock wave is then possible only for Mach numbers such that $\mathcal{D}_{-}^{1}<0$ and $\mathcal{D}_{+}^{2}>0$. It is 


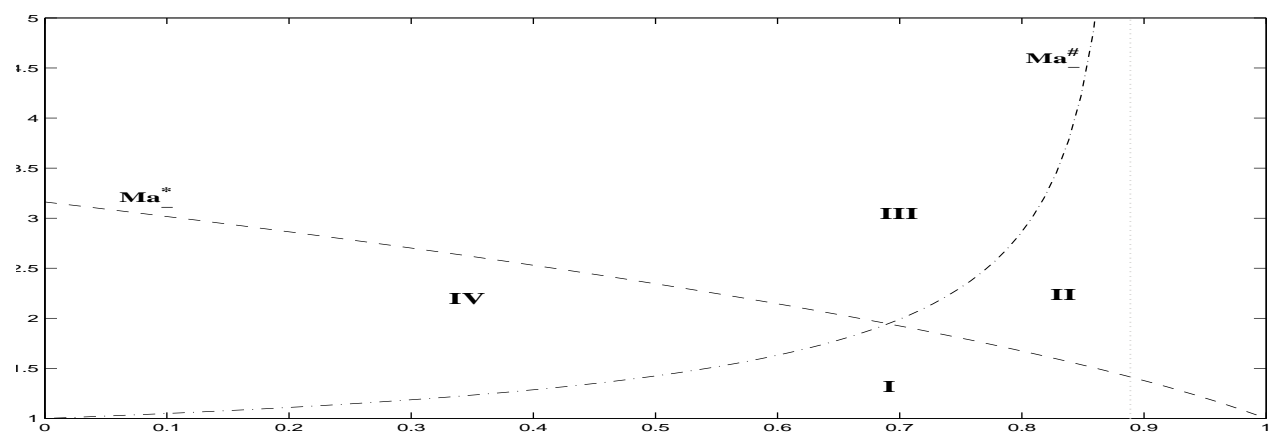

Figure 1: Bifurcation values $\mathrm{Ma}_{-}^{*}$ and $\mathrm{Ma}_{-}^{\sharp}$ versus concentration $c$, for mass ratio $\alpha=0.1$. A smooth shock is admissible in region $I$, a weak solution with one jump characterizes regions $I I$ and $I V$, while in region $I I I$ we may build up a weak solution with two jumps.

clear that $\mathcal{D}_{-}^{1}<0$ only for $\mathrm{Ma}_{-}^{2}<\left(\mathrm{Ma}_{-}^{*}\right)^{2}=\gamma / \alpha$. As concerns $\mathcal{D}_{+}^{2}$, if $\gamma<1 / 5$ then it is positive for any $\mathrm{Ma}_{-}$, otherwise it is positive only for $\mathrm{Ma}_{-}^{2}<\left(\mathrm{Ma}_{-}^{\sharp}\right)^{2}=(\gamma+3) /(5 \gamma-1)$. It can be checked by direct computations that $\mathrm{Ma}_{-}^{*}<\mathrm{Ma}_{-}^{\sharp}$ for

$$
c>c^{*}=\frac{1}{10(1-\alpha)}\left[9-\alpha-\sqrt{\alpha^{2}+62 \alpha+1}\right] .
$$

In conclusion, we have the following cases:

- if $\alpha>\frac{1}{5}$, setting $\mathrm{m}=\min \left\{\mathrm{Ma}_{-}^{*}, \mathrm{Ma}_{-}^{\sharp}\right\}$ and $\overline{\mathrm{m}}=\max \left\{\mathrm{Ma}_{-}^{*}, \mathrm{Ma}_{-}^{\sharp}\right\}$, a smooth solution is allowed for $1<\mathrm{Ma}_{-}<\mathrm{m}$, a solution with one jump is possible for $\mathrm{m}<\mathrm{Ma}_{-}<\overline{\mathrm{m}}$, while for $\mathrm{Ma}_{-}>\overline{\mathrm{m}}$ two jumps are required in order to overcome singularities of both denominators $\mathcal{D}^{1}$ and $\mathcal{D}^{2}$;

- if $\alpha<\frac{1}{5}$, this scenario does not change as long as $c<\bar{c}=4 /[5(1-\alpha)]$, whereas for $\bar{c}<c<1$ a smooth solution is allowed for $1<\mathrm{Ma}_{-}<\mathrm{Ma}_{-}^{*}$, otherwise we may look only for a weak solution with a jump discontinuity.

Bifurcation values for upstream Mach number versus concentration are illustrated in Fig.1, where $\alpha=0.1, \bar{c}=0 . \overline{8}$, and $c^{*}=0.6905$.

\section{Discussion}

This analysis turns out to be consistent with investigation of stability of the two limiting equilibria, performed with the help of symbolic manipulation. Eigenvalues of the Jacobian matrix of system (13) have been evaluated at the downstream and upstream states. Both equilibria obviously admit a vanishing eigenvalue, since equations (13) reproduce the fourth conservation law in (8). The results following from the numerical investigation of the several cases that have been run seem to indicate the following scenario for the 
remaining two eigenvalues. In the upstream state one eigenvalue is positive and the other is negative for $\mathrm{Ma}_{-}<\mathrm{Ma}_{-}^{*}$, while they become both negative for $\mathrm{Ma}_{-}>\mathrm{Ma}_{-}^{*}$. In the downstream equilibrium eigenvalues are both negative for $\mathrm{Ma}_{-}<\mathrm{Ma}_{-}^{\sharp}$, when $\mathrm{Ma}_{-}^{\sharp}$ exists, while one of them becomes positive for $\mathrm{Ma}_{-}>\mathrm{Ma}_{-}^{\sharp}$.

Therefore, according to this scenario, for $\mathrm{Ma}_{-}<\mathrm{m}$ (region $I$ in Fig.1) the downstream equilibrium is asymptotically stable, while the upstream one is a saddle with a one-dimensional unstable manifold. So, the only way to reach it is following the unstable manifold tangent to the one-dimensional upstream unstable eigenspace, getting then attracted by the stable downstream state, which provides the sought smooth shock solution. For all cases run with $\mathrm{Ma}_{-}^{*}<\mathrm{Ma}_{-}^{\sharp}$, both equilibria are asymptotically stable for $\mathrm{Ma}_{-}^{*}<\mathrm{Ma}_{-}<\mathrm{Ma}_{-}^{\sharp}$ (region II in Fig.1), thus no smooth solution connecting the two limiting states is allowed. In particular, the only admissible solution satisfying the upstream condition is the constant solution, and the only way to reach the downstream point is through a suitable jump discontinuity fulfilling the conservation laws (8); a smooth trajectory may then join the point after the jump to the state at $x \rightarrow+\infty$. On the other hand, in all cases that have been checked, if $\mathrm{Ma}_{-}^{\sharp}<\mathrm{Ma}_{-}^{*}$, both equilibria are saddle points for $\mathrm{Ma}_{-}^{\sharp}<\mathrm{Ma}_{-}<\mathrm{Ma}_{-}^{*}$ (region $I V$ in Fig.1); the existence of a smooth solution is prevented because of singularities in (13) analyzed above, but it might be possible to find a jump, consistent with the conservation laws of the model, in which the solution moves from the unstable manifold of the upstream equilibrium to the stable manifold of the downstream point. Finally, for $\mathrm{Ma}_{-}>\overline{\mathrm{m}}$ (region III in Fig.1), in all considered cases the upstream equilibrium is stable while the downstream one is a saddle. The solution starting from the upstream point is again constant, then a jump discontinuity is necessary, but the tail after the jump can not reach the downstream state in a smooth way; once more it might be possible that the tail undergoes a further discontinuity leading eventually the solution to the limiting equilibrium by a second tail on its stable manifold.

A mathematical investigation of the delicate questions arising especially in regions $I I I$ and $I V$ is left as future work. We shall present here only a sample of numerical simulations confirming the previous conjectures. We remark that, for weak solutions with discontinuity in some point $x=x^{0}$, we have to look for piecewise smooth solutions whose limits for $x \rightarrow x_{-}^{0}$ and $x \rightarrow x_{+}^{0}$ fulfill the constraints following from (4). Since the contributions $R_{12}$ and $S_{12}$ on the right hand sides are bounded functions, those equations imply continuity of the quantities under derivative operator across the jump. If we know the configuration on one side of the jump (whose fields will be labelled by subscript $*$ ), the corresponding one on the other side, denoted with subscript $\bullet$, is provided by

$$
\begin{aligned}
\rho_{\bullet}^{1}\left(u_{\bullet}^{1}\right)^{2}+n_{\bullet}^{1} T_{\bullet}^{1} & =k_{1}^{A} \\
\rho_{\bullet}^{1}\left(u_{\bullet}^{1}\right)^{3}+5 n_{\bullet}^{1} T_{\bullet}^{1} u_{\bullet}^{1} & =k_{1}^{B} \\
\rho_{\bullet}^{2}\left(u_{\bullet}^{2}\right)^{3}+5 n_{\bullet}^{2} T_{\bullet}^{2} u_{\bullet}^{2} & =k_{2}^{B}
\end{aligned}
$$

where $k_{s}^{A}:=\rho_{*}^{s}\left(u_{*}^{s}\right)^{2}+n_{*}^{s} T_{*}^{s}$ and $k_{s}^{B}:=\rho_{*}^{s}\left(u_{*}^{s}\right)^{3}+5 n_{*}^{s} T_{*}^{s} u_{*}^{s}$. Appropriate manipulations on equations (17) lead first to the algebraic equation for $u_{\bullet}^{1}$

$$
4 \rho_{*}^{1} u_{*}^{1}\left(u_{\bullet}^{1}\right)^{2}-5 k_{1}^{A} u_{\bullet}^{1}+k_{1}^{B}=0
$$


with solutions

$$
u_{\bullet}^{1}=u_{*}^{1}, \quad \text { or } \quad u_{\bullet}^{1}=\frac{\rho_{*}^{1}\left(u_{*}^{1}\right)^{2}+5 n_{*}^{1} T_{*}^{1}}{4 \rho_{*}^{1} u_{*}^{1}}=: \tilde{u}^{1},
$$

then to the expressions for temperatures

$$
T_{\bullet}^{1}=\frac{k_{1}^{B}-k_{1}^{A} u_{\bullet}^{1}}{4 n_{*}^{1} u_{*}^{1}}, \quad T_{\bullet}^{2}=\frac{k_{2}^{B}-\rho_{*}^{2} u_{*}^{2}\left(u_{\bullet}^{2}\right)^{2}}{5 n_{*}^{2} u_{*}^{2}},
$$

and finally to a quadratic equation analogous to (18) for $u_{\bullet}^{2}$, with roots

$$
u_{\bullet}^{2}=u_{*}^{2}, \quad \text { or } \quad u_{\bullet}^{2}=\frac{\rho_{*}^{2}\left(u_{*}^{2}\right)^{2}+5 n_{*}^{2} T_{*}^{2}}{4 \rho_{*}^{2} u_{*}^{2}}=: \tilde{u}^{2} .
$$

There are thus four possible outputs for velocities after the jump, namely $\left(u_{*}^{1}, u_{*}^{2}\right),\left(u_{*}^{1}, \tilde{u}^{2}\right)$, $\left(\tilde{u}^{1}, u_{*}^{2}\right)$, and $\left(\tilde{u}^{1}, \tilde{u}^{2}\right)$ (the first, however, must be obviously discarded). A discontinuity necessarily exists due to the denominator(s) that would be bound to vanish if the solution were continuous, but occurrence of other, even several, jumps may not be excluded at this point. Similarly, one can not exclude simultaneous vanishing of both denominators at some point. All other species parameters after the jump are determined by the knowledge of velocities $u_{\bullet}^{s}$ (expressions are omitted here for brevity). In particular, they are continuous across the jump for the species whose velocity is continuous. For any species undergoing discontinuity it is interesting to notice that

$$
\begin{aligned}
\mathcal{D}_{\bullet}^{s} & =3 \rho_{*}^{s} u_{*}^{s} u_{\bullet}^{s}-5 \frac{n_{*}^{s} u_{*}^{s} T_{\bullet}^{s}}{u_{\bullet}^{s}}=-\frac{1}{u_{\bullet}^{s}}\left[3 \rho_{*}^{s}\left(u_{*}^{s}\right)^{2}-5 n_{*}^{s} T_{*}^{s}\right] \frac{\rho_{*}^{s}\left(u_{*}^{s}\right)^{2}+5 n_{*}^{s} T_{*}^{s}}{4 \rho_{*}^{s} u_{*}^{s}} \\
& =-\left[3 \rho_{*}^{s}\left(u_{*}^{s}\right)^{2}-5 n_{*}^{s} T_{*}^{s}\right]=-\mathcal{D}_{*}^{s},
\end{aligned}
$$

namely the relevant denominator changes sign keeping the same magnitude.

\section{Numerical results}

We shall show in this section the shock profiles resulting from the numerical integration of system (13) for the fields $u^{1}, u^{2}, \theta$. Smooth solutions are normalized in such a way that $u^{1}(0)=\left(u_{+}+u_{-}\right) / 2$, whereas for weak solutions with only one jump, this is localized at $x=0$. All computations were performed by integrating via standard Runge-Kutta methods.

As reference case, we consider the mixture of Helium (species 1) and Argon (species 2) considered in [8]. The dimensionless masses are taken to be $m^{1}=4, m^{2}=40$, so that mass ratio follows as $\alpha=0.1$. Moreover, we will keep fixed the following upstream parameters $n_{-}^{1}=0.753, n_{-}^{2}=0.247, u_{-}=1.63$ (thus $n_{-}=1$ and $c=0.753$ ) while $T_{-}$ will be changed in the various plots, in order to increase the upstream Mach number $\mathrm{Ma}_{-}$. For the bifurcations values of the Mach number we get $\mathrm{Ma}_{-}^{*}=1.7953, \mathrm{Ma}_{-}^{\sharp}=2.3309$. When $\mathrm{Ma}_{-}$is less than both bifurcation values, a smooth shock solution is admissible, and can be found numerically by choosing an initial point in a close neighborhood of the 

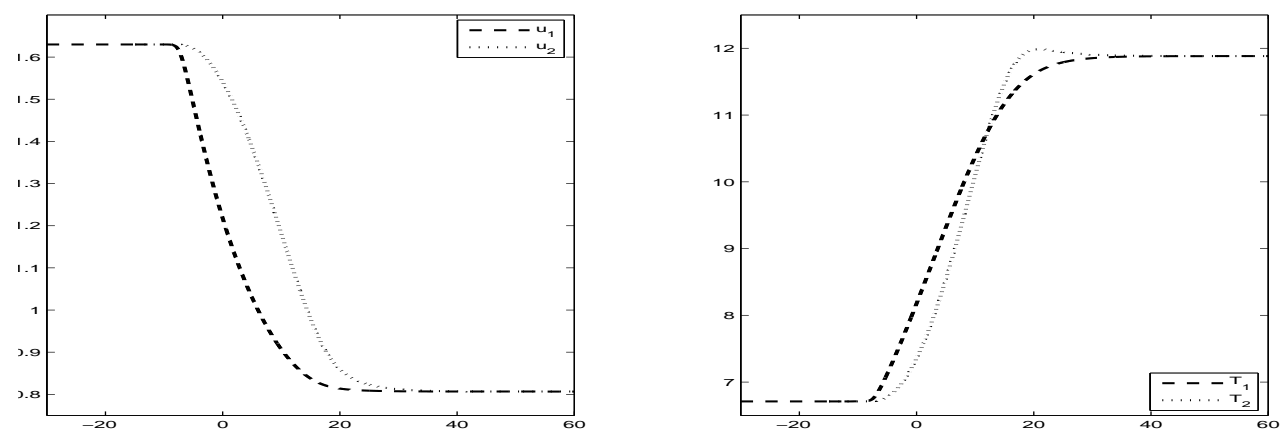

Figure 2: Velocity and temperature profiles when $c=0.753$ and $\mathrm{Ma}_{-}=1.75$ (region I)

upstream equilibrium, in the direction of its one-dimensional unstable eigenspace. If $\mathrm{Ma}_{-}$ is close to 1 , differences between velocities $u^{1}, u^{2}$ and temperatures $T^{1}, T^{2}$ along the shock are hardly visible on the plots (not shown here), but they increase for increasing $\mathrm{Ma}_{-}$.

When we increase $\mathrm{Ma}_{-}$to 1.75 , close to the lower bifurcation value, but still in the "smooth" region, the situation looks like in Fig.2. In this case, $T_{-}=6.7107, n_{+}^{1}=$ $1.5215, n_{+}^{2}=0.4991, u_{+}=0.8067, T_{+}=11.8834$, and the figure shows a fast detachment from an almost constant trend upstream, and occurrence of overshooting in one species temperature. Notice that there is a point during the evolution in which $T^{1}=T^{2}$ (but $u^{1} \neq u^{2}$ ), then the two species temperatures split again before reaching the common equilibrium value $T_{+}$.

If we now increase $\mathrm{Ma}_{-}$across the first bifurcation value, taking $\mathrm{Ma}_{-}^{*}<\mathrm{Ma}_{-}<\mathrm{Ma}_{-}^{\sharp}$, the existence of a smooth solution is prevented since a singularity of system (13) enters the phase space, so we are led to look for a possible weak solution with one jump discontinuity. As discussed above, the upstream equilibrium is here asymptotically stable, so the unique way to fulfill the upstream condition is by constant profiles, with $u^{1}=u^{2}=u_{-}$and $T^{1}=T^{2}=T_{-}$. We recall that in this region the denominator $\mathcal{D}^{2}$ is positive both in the upstream and downstream equilibria, while the sign of $\mathcal{D}^{1}$ changes from positive (for $x \rightarrow-\infty$ ) to negative (for $x \rightarrow+\infty$ ). Also, according to (22), any discontinuity in the species $s$ is accompanied by a change of sign of the denominator $\mathcal{D}^{s}$. Among the three possible choices of velocities $\left(u_{\bullet}^{1}, u_{\bullet}^{2}\right)$ for a jump compatible with conservation laws, the only one that changes the sign of $\mathcal{D}^{1}$ and not of $\mathcal{D}^{2}$ is the one in which $u^{2}$ remains continuous (equal to $u_{-}$) while $u^{1}$ jumps to $\frac{\rho_{-}^{1}\left(u_{-}\right)^{2}+5 n_{-}^{1} T_{-}}{4 \rho_{-}^{1} u_{-}}$. After this discontinuity the solution exhibits a tail that is attracted in a smooth way by the stable downstream limiting point. The trend is shown in Fig.3, relevant to $\mathrm{Ma}_{-}=2$, corresponding to $T_{-}=5.1379, n_{+}^{1}=1.7211, n_{+}^{2}=0.5646, u_{+}=0.7131, T_{+}=10.6772$.

By increasing further $\mathrm{Ma}_{-}$, crossing also the second bifurcation value $\mathrm{Ma}_{-}^{\sharp}$, singularities of both denominators $\mathcal{D}^{1}$ and $\mathcal{D}^{2}$ enter the phase space. Again the constant profile is the unique solution compatible with the stable upstream equilibrium, but now the situation gets more involved. Numerical simulations show that after the unique jump that would change simultaneously the sign of both denominators (corresponding to discontinuity of 

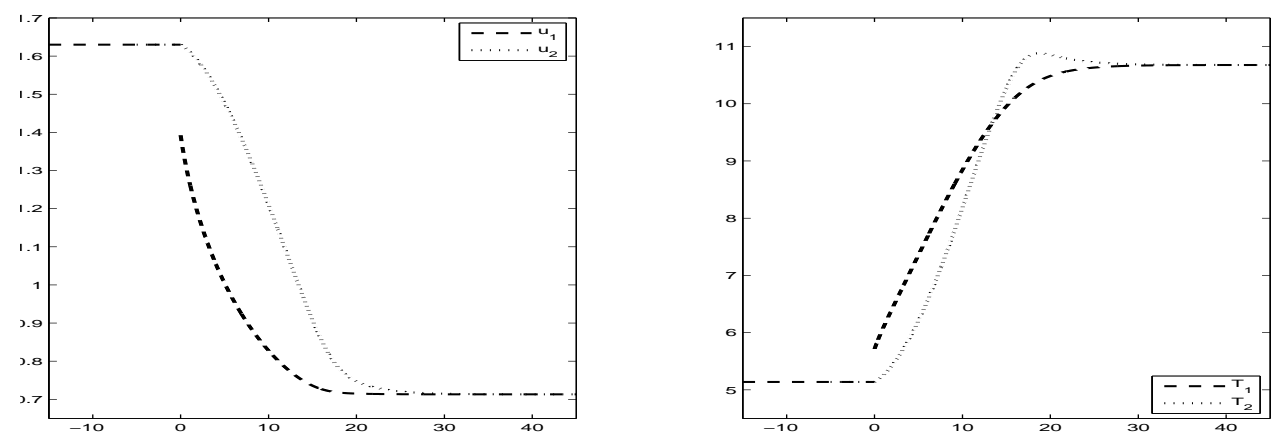

Figure 3: Velocity and temperature profiles when $c=0.753$ and $\mathrm{Ma}_{-}=2$ (region II)
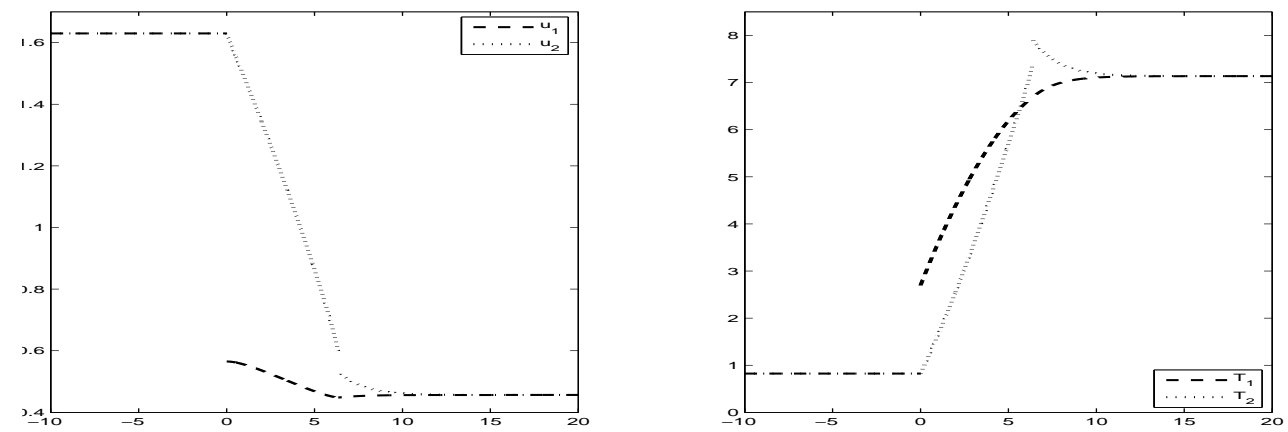

Figure 4: Velocity and temperature profiles when $c=0.753$ and $\mathrm{Ma}_{-}=5$ (region III)

both velocities), evolution does not enter into the stable manifold of the downstream point (now a saddle), thus no weak shock solution with only one jump seems to be allowed. However, we are able to build up a weak solution with two discontinuities. Specifically, we allow a first discontinuity with the same features of the previous case (same type of jump for $u^{1}$ and $T^{1}$, no jump for species 2) and consider the tail originating from it. On the other hand, we move backward from $+\infty$ starting tangent to its stable manifold. We realize that there is exactly one pair of points $\left(u_{\bullet}^{1}, u_{\bullet}^{2}, \theta_{\bullet}\right)$ (belonging to the solution after the first jump) and $\left(u_{*}^{1}, u_{*}^{2}, \theta_{*}\right)$ (belonging to the stable manifold of $+\infty$ ) that fulfill conservations (17); therefore, a jump between these two points is admissible, and this allows to reach in a compatible way the downstream equilibrium. More precisely, we note that in the second jump only quantities relevant to species 2 are really discontinuous, and the sign of the denominator $\mathcal{D}^{2}$ changes as expected. When Mach number is close to the bifurcation value $\mathrm{Ma}_{-}^{\sharp}$, the second jump discontinuity (for $u^{2}$ and $T^{2}$ ) is hardly visible, but again it enlarges for increasing $\mathrm{Ma}_{-}$. It can be seen clearly in Fig.4 relevant to $\mathrm{Ma}_{-}=5$, for which $T_{-}=0.8221, n_{+}^{1}=2.6893, n_{+}^{2}=0.8821, u_{+}=0.4564, T_{+}=7.1355$.

Finally, let us change the upstream number densities, taking $n_{-}^{1}=0.4$ and $n_{-}^{2}=0.6$, so that concentration becomes $c=0.4$. In this case $\mathrm{Ma}_{-}^{\sharp}=1.2863, \mathrm{Ma}_{-}^{*}=2.5298$, and we focus our attention on cases with Mach number such that $\mathrm{Ma}_{-}^{\sharp}<\mathrm{Ma}_{-}<\mathrm{Ma}_{-}^{*}$ for which both equilibria are saddle points, and at least one jump exists, due to $\mathcal{D}^{2}$. 

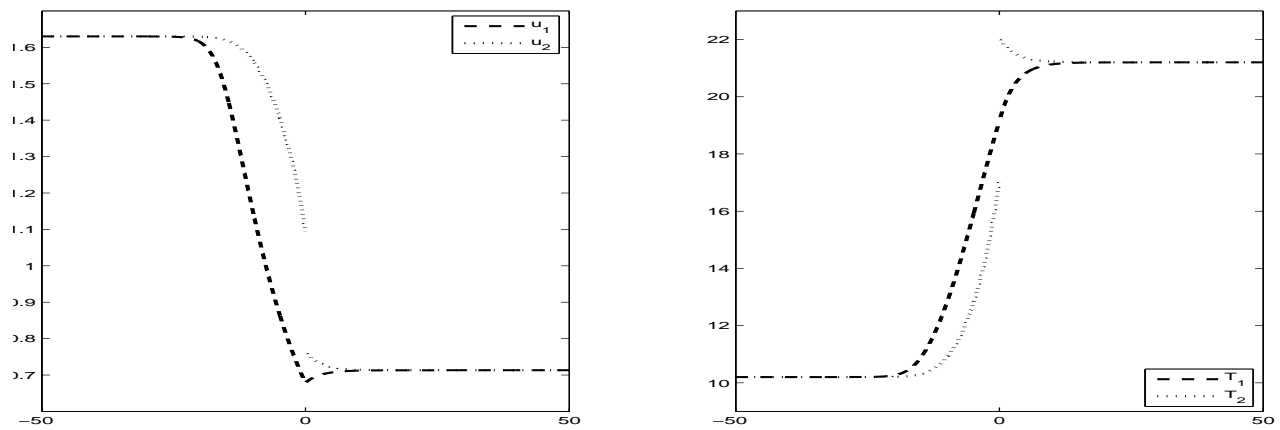

Figure 5: Velocity and temperature profiles when $c=0.4$ and $\mathrm{Ma}_{-}=2$ (region IV)

The situation is again involved; however, a (unique) pair of compatible points may be found if we test (17) using on one side the stable downstream manifold (in the backward direction), and on the other side the solution tangent to the upstream unstable manifold (in the forward direction). We obtain in this way a weak shock solution with one jump, where only species 2 is discontinuous. This is shown in Fig.5, relevant to $\mathrm{Ma}_{-}=2$, with $T_{-}=10.2025, n_{+}^{1}=0.9143, n_{+}^{2}=1.3714, u_{+}=0.7131, T_{+}=21.2021$.

\section{Acknowledgements}

Fruitful discussions with F. Conforto and S. Simic on the subject of the present work are gratefully acknowledged.

\section{References}

[1] Bisi, M., Martalò, G., Spiga, G.: Multi-temperature hydrodynamic limit from kinetic theory in a mixture of rarefied gases. Acta Appl. Math. 122, 37-51 (2012).

[2] Bisi, M., Martalò, G., Spiga, G.: Multi-temperature fluid-dynamic model equations from kinetic theory in a reactive gas: the steady shock problem. Comput. Math. Appl. 66, 1403-1417 (2013).

[3] Boillat, G., Ruggeri, T.: On the shock structure problem for hyperbolic system of balance laws and convex entropy. Continuum Mech. Thermodyn. 10, 285-292 (1998).

[4] Cercignani, C.: Rarefied Gas Dynamics. From Basic Concepts to Actual Calculations. University Press, Cambridge (2000).

[5] Currò, C., Fusco, D.: Discontinuous travelling wave solutions for a class of dissipative hyperbolic models. Rend. Mat. Acc. Lincei, s. 6 16, 61-71 (2005).

[6] Groppi, M., Spiga, G., Takata, S.: The steady shock problem in reactive gas mixtures. Bullet. Inst. Math. Acad. Sinica (New Series) 2, 935-956 (2007). 
[7] Groppi, M., Spiga, G., Zus, F.: Euler closure of the Boltzmann equations for resonant bimolecular reactions. Phys. Fluids 18, 057105 (2006).

[8] Madjarevic, D., Simic, S.: Shock structure in Helium-Argon mixture - A comparison of hyperbolic multi-temperature model with experiment. Europhys. Lett. 102, 44002 (2013).

[9] Müller, I., Ruggeri, T.: Rational Extended Thermodynamics. Springer, New York (1988).

[10] Ruggeri, T., Simic, S.: Average temperature and Maxwellian iteration in multitemperature mixtures of fluids. Phys. Rev. E 80, 026317 (2009). 